Sādhanā Vol. 39, Part 5, October 2014, pp. 1227-1244. (C) Indian Academy of Sciences

\title{
Impact of pipes networks simplification on water hammer phenomenon
}

\author{
ALI A M GAD ${ }^{1}$ and HASSAN I MOHAMMED ${ }^{1,2, *}$ \\ ${ }^{1}$ Civil Engineering Department, Assiut University, Assiut 71516, Egypt \\ ${ }^{2}$ Civil and Environmental Engineering Department, Majmaah University, \\ Al Majmaah 11952, Saudi Arabia \\ e-mail: hassanmohamed_2000@yahoo.com; aligad64@yahoo.com
}

MS received 9 July 2013; revised 28 February 2014; accepted 14 March 2014

\begin{abstract}
Simplification of water supply networks is an indispensible design step to make the original network easier to be analysed. The impact of networks' simplification on water hammer phenomenon is investigated. This study uses two loops network with different diameters, thicknesses, and roughness coefficients. The network is fed from a boundary head reservoir and loaded by either distributed or concentrated boundary water demands. According to both hydraulic and hydraulic plus water quality equivalence, three simplification levels are performed. The effect of demands' concentration on the transient flow is checked. The transient flow is initialized by either concentrated or distributed boundary demands which are suddenly shut-off or released. WHAMO software is used for simulation. All scenarios showed that both hydraulic equivalence and demands' concentration simplifications increase the transient pressure and flow rate. However, hydraulic plus water quality equivalence simplification produces an adverse effect. Therefore, simplifications of the networks should be done carefully. Also, it was found that pump shut-off gives the same trend of valve shut-off or release.
\end{abstract}

Keywords. Water hammer; pipes network; simplification; demands variations.

\section{Introduction}

The potable water distribution system is one of the most significant hydraulic engineering accomplishments. Potable water can be delivered to water users through distribution systems. However, variable water demands and water usage patterns can produce significant variations of pressure in the distribution system, especially when the changes are sudden. Sudden changes

${ }^{*}$ For correspondence 
of water demands can create transient flow that could make so many undesirable consequences such as backflow, negative pressure, or excessive high pressure. Therefore, it is important for engineers to explore the various transient flow effects and to develop the emergency response strategies in order to minimize the negative impacts (Kwon 2007). The total force acting within a pipe is obtained by summing the steady-state and transient pressures in the line. The severity of transient pressures must be accurately determined so that water mains can be properly designed to withstand these additional loads (Jung et al 2007). Many researchers studied the water hammer phenomenon in the last decades with different viewpoints. For example, Abd El-Gawad (1994), Ali et al (2010), Jönnsson (1999), Stephenson (2002), Yang (2001), and many others.

Al-Khomairi (2005) discussed the use of the steady-state orifice equation for the computation of unsteady leak rates from pipes through crack or rupture. It has been found that the orifice equation gives a very good estimation of the unsteady leak rate history for normal leak openings. Fouzi \& Ali (2001) studied water hammer in gravity piping systems due to sudden closure of valves, using both the most effective numerical methods for discretizing and solving the problem; the finite difference method using water hammer and mass oscillation (WHAMO) program and the method of characteristics with software AFT impulse. They showed that pressure fluctuations vary dangerously especially in the case of pipes which has variable characteristics (section changes with a divergence, a convergence or a bifurcation). Jung et al (2009) studied the effect of pressure-sensitive demand on transient pressure. They concluded that a pressuresensitive demand formulation should be used for surge analysis to adequately evaluate both system performance and the ultimate cost of system protection.

Mohamed (2003) introduced the effect of different parameters such as time of valve closure, pipes' material rigidity, and pipes roughness on the transient pressure damping. It was found that the pipe friction factor and the closing time of the valve have a significant effect on the transient pressure reduction and the elastic pipes such as PVC are better than rigid pipes in pressure damping.

Ramos et al (2004) carried out several simulations and experimental tests in order to analyse the dynamic response of single pipelines with different characteristics, such as pipes' material, diameters, thicknesses, lengths and transient conditions. They concluded that being the plastic pipe with a future increasing application, the viscoelastic effect must be considered, either for model calibration, leakage detection or in the prediction of operational conditions (e.g., start up or trip-off electromechanical equipment, valve closure or opening).

Samani \& Khayatzadeh (2002) employed the method of characteristics to analyse transient flow in pipe networks. They applied various numerical tests to examine the accuracy of these methods and found that the method in which the implicit finite difference was coupled with the method of characteristics to obtain the discretized equations is the best when compared to others.

According to the aforementioned studies, water hammer in pipes networks has been studied from different viewpoints. However, each water supply network has its own special characteristics which make it different from other networks. Also, due to the lack of field measurements which are costly, it becomes important to use numerical models to gain an understanding about the behaviour of networks under transient effects.

This study aims to investigate the effect of the hydraulic equivalence, hydraulic plus water quality equivalence, and demands' concentration simplifications of pipes networks on the transient pressure head and flow rate induced from sudden demands shut-off or release. 


\section{Theoretical considerations}

Because of the difficulty in solution of water hammer governing equations, engineers in pipelines design may neglect this phenomenon. Recently a number of numerical methods which may be used to solve these equations and suitable for digital computer analyses have been reported in the literature (Chaudhry \& Yevjevich 1981).

\subsection{Governing equations for unsteady flow in pipelines}

The governing equations for unsteady flow in pipeline are derived under the following assumptions including; (1) one-dimensional flow i.e., velocity and pressure are assumed constant at a cross section; (2) the pipe is full and remains full during the transient; (3) no column separation occurs during the transient; (4) the pipe wall and fluid behave linearly elastically; and (5) unsteady friction loss is approximated by steady-state losses.

The unsteady flow inside the pipeline is described in terms of unsteady mass balance (continuity) equation and unsteady momentum equation, which define the state of variables of $V$ (velocity) and P (pressure) given as Simpson \& Wu (1997);

$$
\begin{gathered}
\frac{\partial \rho}{\partial t}+V \frac{\partial \rho}{\partial x}+\rho \frac{\partial V}{\partial x}+\frac{\rho}{A} \frac{d A}{d t}=0, \\
\frac{\partial V}{\partial t}+V \frac{\partial V}{\partial x}+\frac{1}{\rho} \frac{\partial P}{\partial x}-g \sin \alpha+\frac{f|V| V}{2 D}=0,
\end{gathered}
$$

where $x=$ distance along the pipeline; $t=$ time; $V=$ velocity; $P=$ hydraulic pressure in the pipe; $g=$ acceleration due to gravity; $f=$ Darcy-Weisbach friction factor; $\rho=$ fluid density; $D=$ pipe diameter; $\alpha=$ pipe slope angle, and $A=$ cross sectional area of the pipe.

Eq. (1) is the continuity equation and takes into account the compressibility of water and the flexibility of pipe material. Eq. (2) is the equation of motion. In Eq. (1), the terms $1 / \rho\left(\frac{\partial \rho}{\partial t}+\frac{\partial \rho}{\partial x} V\right)$ are replaced by equivalent $1 / \rho d \rho / d t$, where $V=d x / d t, \frac{d \rho}{d t}=\rho / K d P / d t$, and $K$ is the bulk modulus of the fluid. Also, the fourth term in Eq. (1) can be expressed as $\left(1-v^{2}\right) \rho D / e E d P / d t$, where $v$ is the poison's ratio of the pipe, $e$ is the pipe wall thickness and $E$ is the Young's modulus of elasticity of the pipe. Substitution by these abbreviations in Eq. (1), it can be reduced to the following formula;

$$
\frac{d P}{d t}\left[\frac{1}{K}+\left(\frac{1-v^{2}}{E}\right) \frac{D}{e}\right]+\frac{\partial V}{\partial x}=0 .
$$

Wave speed can be defined as the time taken by the pressure wave generated by instantaneous change in velocity to propagate from one point to another in a closed conduit. Wave speed (c) can be expressed as;

$$
\frac{1}{\rho c^{2}}=\left[\frac{1}{K}+\left(\frac{1-v^{2}}{E}\right) \frac{D}{e}\right]=\frac{1}{K}\left[1+\frac{K c_{1} D}{E e}\right],
$$

where: $c_{1}=\left(1-v^{2}\right)$. Substitution by Eq. (4) in (3) and dividing the result by $\gamma$ yields;

$$
\left[\frac{\partial H}{\partial t}+\frac{\partial H}{\partial x} V\right]+\frac{c^{2}}{g} \frac{\partial V}{\partial x}=0
$$


where $H$ is the piezometric head, i.e., pressure head plus the elevation head. The term $\partial H / \partial x$ is small compared to $\partial H / \partial t$ and it is often neglected. Thus, the simplified form of the continuity equation in terms of discharge, Eq. (5) becomes

$$
\frac{\partial H}{\partial t}+\frac{\partial Q}{\partial x} \frac{c^{2}}{g A}=0 .
$$

By the same way, the momentum equation, i.e., Eq. (2) can be simplified and written in terms of discharge and piezometric head as follows

$$
\frac{\partial H}{\partial x}+\frac{1}{g A} \frac{\partial Q}{\partial t}+\frac{f Q|Q|}{2 g D A^{2}}=0.0 .
$$

\subsection{Implicit finite difference solution method}

The continuity and momentum equations form a pair of hyperbolic, partial differential for which an exact solution cannot be obtained analytically. However, other methods have been developed to solve water hammer equations. If the equations are hyperbolic, it means the solutions follow certain characteristic pathways. For water hammer equations, the wave speed is the characteristic. The implicit finite difference method is a numerical method used for solving water hammer equations. The implicit method replaces the partial derivatives with finite differences and provides a set of equations that can then be solved simultaneously. The computer program WHAMO uses the implicit finite-difference technique but converts its equations to a linear form before it solves the set of equations (Fitzgerald \& Van Blaricum 1998).

The solution space is discretized into the $x-t$ plane, so that at any point on the grid $(x, t)$ there is a certain $\mathrm{H}$ and $\mathrm{Q}$ for that point, $H(x, t)$ and $Q(x, t)$ as shown in figure 1.

The momentum equation and the continuity equation can be represented in a short form by introducing the following coefficients for the known values in a system;

$$
\begin{gathered}
\alpha_{j}=\frac{2 \Delta t c_{j}^{2} \theta}{g A_{j} \Delta x_{j}}, \\
\beta_{j}=\left(H_{j+1}^{n}+H_{j}^{n}\right)+\frac{(1-\theta)}{\theta} \alpha_{j}\left(Q_{j}^{n}-Q_{j+1}^{n}\right),
\end{gathered}
$$

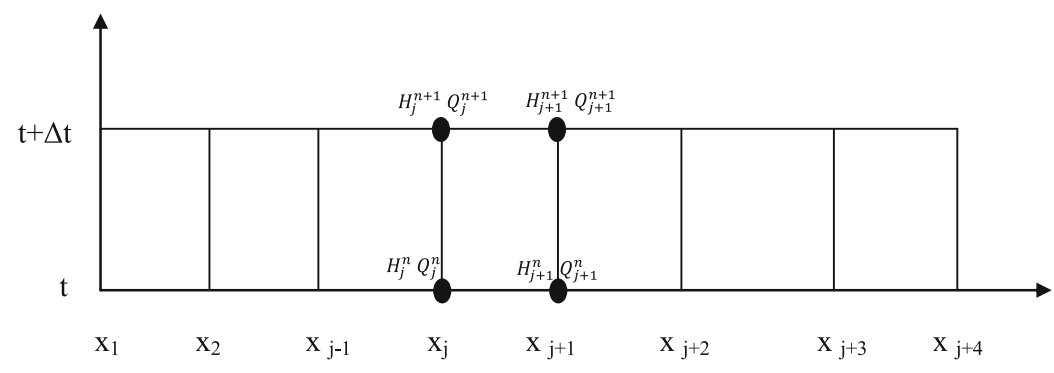

Figure 1. The finite difference grid. 


$$
\begin{gathered}
\gamma_{j}=\frac{\Delta x_{j}}{2 g \theta A_{j} \Delta t}, \\
\delta_{j}=\frac{(1-\theta)}{\theta}\left(H_{j}^{n}-H_{j+1}^{n}\right)+\gamma_{j}\left(Q_{j}^{n}+Q_{j+1}^{n}\right)-\frac{\Delta x_{j} f_{j}}{4 g \theta D_{j} A_{j}^{2}}\left(Q_{j}^{n}\left|Q_{j}^{n}\right|+Q_{j+1}^{n}\left|Q_{j+1}^{n}\right|\right),
\end{gathered}
$$

where $\theta$ is a weighing factor included for numerical stability. All parameters for the coefficients should be known from the properties of the pipe or the values of head and flow at the previous time step. With the coefficients, the momentum and continuity equations of the $j^{\text {th }}$ segment of the pipe become as given by Batterton (2006) as follows;

$$
\begin{gathered}
\text { Momentum : }-H_{j+1}^{n}+H_{j+1}^{n+1}+\gamma_{j}\left(Q_{j}^{n+1}+Q_{j+1}^{n+1}\right)=\delta_{j}, \\
\text { Continuity : } H_{j+1}^{n}+H_{j+1}^{n+1}+\alpha_{j}\left(Q_{j+1}^{n+1}-Q_{j}^{n+1}\right)=\beta_{j} .
\end{gathered}
$$

Now, with equations for the all links and nodes in the system, the initial and boundary conditions, a matrix of the linear system of equations can be set-up to solve for head and flow everywhere, simultaneously, for the first time step. The process is repeated for the next time step, and again for the next step until the specified end of the simulation.

\section{Applications}

The simple pipes network shown in figure 2 consisting of 11 joints $(\mathrm{J} 1 \sim \mathrm{J} 11)$ and 12 high density polyethylene (HDPE) pipes $(\mathrm{C} 1 \sim \mathrm{C} 12)$ at the same elevation is representative of a general parallel/series system. HDPE pipes with their common low Young's modulus were preferred in this application to avoid negative pressure waves to drop to the saturated vapor pressure of the water which form a cavity in the fluid as the simulation program (WHAMO) does not allow for the effect of cavitations. Other strong pipes networks with high Young's modulus must employ systems to help control increase and decrease in pressure due to water hammer. The Young's modulus for the HDPE material and water were taken as 0.80 and $2.20 \mathrm{GPa}$, respectively. Joint $\mathrm{J} 10$ only has a boundary concentrated demand of $126 \mathrm{~L} / \mathrm{s}$ and the network is fed by a reservoir

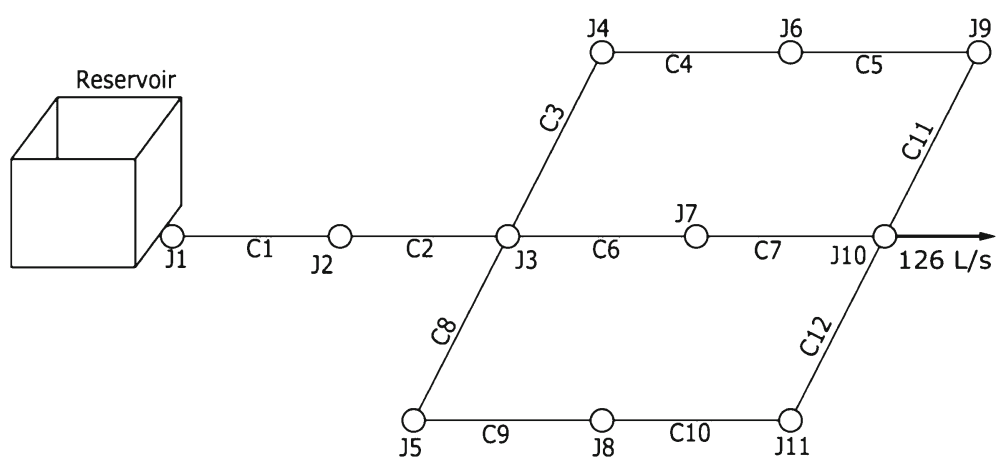

Figure 2. A Simple pipes network (the original network). 
with a boundary head of $59 \mathrm{~m}$. Each pipe from $\mathrm{C} 1$ to $\mathrm{C} 12$ has a circular cross section. Table 1 gives lengths, diameters and Darcy-Weisbach friction factors $(f)$ for all pipes in the network. The thicknesses of the pipes' walls were taken according to their diameters to suit for a working pressure of 10 bars.

\section{Simplification methods}

\subsection{The hydraulic equivalence simplification method}

Using conservation of energy across a set of pipes in parallel or series, equivalent pipes relationships can be derived. Since these relationships are developed from conservation of energy, the equivalent pipes have consistent flow and pressure losses as the original set of pipes. Typically, an equivalent diameter is determined by fixing the equivalent pipe's length and roughness, (Mohamed \& Ahmed 2011). Eqs. (14) and (15) can be used for calculating the hydraulic equivalent diameter for $n$ pipes in series and in parallel, respectively.

$$
\frac{1}{D_{e}^{5}}=\sum_{i=1}^{n} \frac{1}{D_{i}^{5}}\left(\frac{f_{i}}{f_{e}}\right) \frac{L_{i}}{L_{e}}
$$

and

$$
D_{e}=\left[\sum_{i=1}^{n}\left(\frac{f_{e}}{f_{i}}\right)^{0.5}\left(\frac{L_{e}}{L_{i}}\right)^{0.5} D_{i}^{2.5}\right]^{0.4}
$$

where $f_{i}, D_{i}$ and $L_{i}$ are the Darcy-Weisbach friction factor, diameter, and length of the pipe $i$ in series or parallel and $f_{e}, \mathrm{D}_{\mathrm{e}}$ and $\mathrm{L}_{\mathrm{e}}$ are the same parameters for the hydraulic equivalent system.

Table 1. Lengths, diameters and friction factors for all pipes of the original network.

\begin{tabular}{lccc}
\hline Pipe ID & Length $(\mathrm{m})$ & Diameter $(\mathrm{mm})$ & $\begin{array}{c}\text { Darcy-Weisbach } \\
\text { friction factor }(f)\end{array}$ \\
\hline C1 & 305 & 300 & 0.026 \\
C2 & 305 & 300 & 0.026 \\
C3 & 305 & 300 & 0.024 \\
C4 & 305 & 200 & 0.020 \\
C5 & 305 & 150 & 0.018 \\
C6 & 305 & 200 & 0.020 \\
C7 & 305 & 150 & 0.018 \\
C8 & 305 & 250 & 0.022 \\
C9 & 305 & 200 & 0.020 \\
C10 & 305 & 150 & 0.018 \\
C11 & 305 & 300 & 0.024 \\
C12 & 305 & 250 & 0.022 \\
\hline
\end{tabular}


By fixing two of the three parameters, the third can be determined using a form of the above equations.

\subsection{The hydraulic and water age equivalence simplification method}

In general, water quality has an adverse relation with its age thus the travel time of water in pipes could be used to indicate its quality. Raczynski et al (2008) developed the following equation for computing the water age equivalent diameter, $D_{\text {ew }}$.

$$
D_{e w}=\left[\frac{\sum_{i=1}^{n}\left(D_{i}^{2} L_{i}\right)}{L_{e}}\right]^{0.5} \text {. }
$$

$D_{e w}$ ensures that the travel time in the equivalent pipe will equal that of the series or parallel pipes. However, it does not ensure that the system will be hydraulically equivalent. Since Eq. (16) shows that $D_{e w}$ is independent on $f$, it is possible to find an equivalent hydraulic system without affecting the travel time equivalence by modifying the pipe roughness. To do so, rather than solving for $D_{e}$ for a defined value of $f_{e}$ in hydraulic equivalence equations Eqs. (14) and (15), $D_{e}$ is set to $D_{e w}$, and $f_{e}$ is solved for as an unknown term.

\subsection{Demands' concentration simplification method}

Simplification is an important primary design step of water supply and irrigation pipes networks. In most real networks, the demands leave through most parts of the pipe lines. However, an indispensible design step of pipes networks is the demands' concentration. The demands that leave the network at distributed locations over its pipes' lengths are replaced with equivalent concentrated demands that reallocated at the joints of the simplified network.

\subsection{Applied simplifications on the used network}

The effect of the equivalence simplification methods are evaluated for the used pipes network with different three levels of simplifications (aggregations orskeletonizations) and demands' concentration. The simplificationis loosely defined as the removing of pipes and nodes from a network to make the model simpler. In this study, the aggregation simplifies the system by replacing a series or parallel set of pipes with a single pipe. The first level of simplification (Level 1) as shown in figure 3a aggregates the two series pipes between nodes (J4 J9), (J3 J10), and (J5 J11) and removes joints J6, J7 and J8. Since there are no demands at these nodes, no demands reallocation are required. The second level of simplification (Level 2) is shown in figure $3 \mathrm{~b}$ which aggregates the upper and lower series pipes between nodes $\mathrm{J} 3$ and $\mathrm{J} 10$ to only one pipe on each upper and lower side. The third level of simplification(level 3) as shown in figure 3c replaces the three parallel pipes of level 2 with a single pipe. Table 2 shows the calculated properties of the pipes of the simplified network for the three levels of simplifications according to both hydraulic and hydraulic plus water quality equivalence. The other simplification type is performed only on the original network, which includes concentrating distributed demands with a value of $14 \mathrm{~L} / \mathrm{s}$ loaded on 9 nodes $(\mathrm{J} 3 \sim \mathrm{J} 11)$ to be at the end node (J10) with a total concentrated equivalent value of $126 \mathrm{~L} / \mathrm{s}$. 


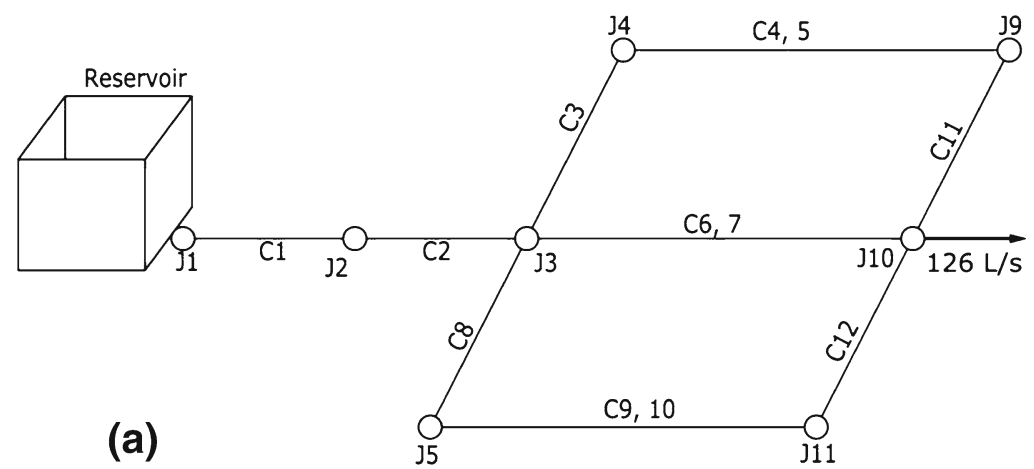

$\mathrm{C} 3,4,5,11$

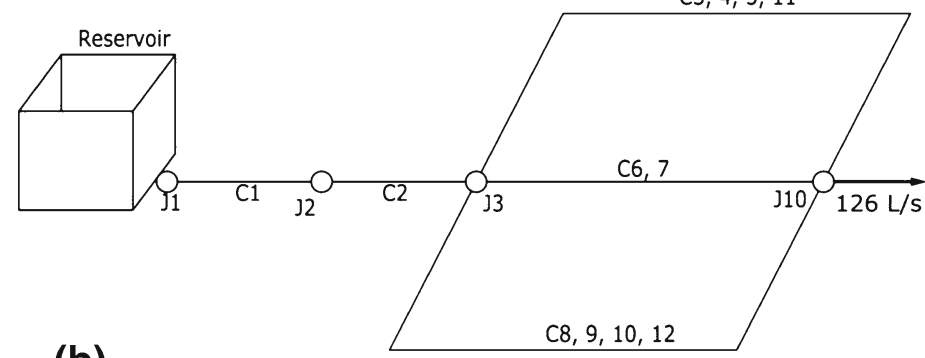

(b)

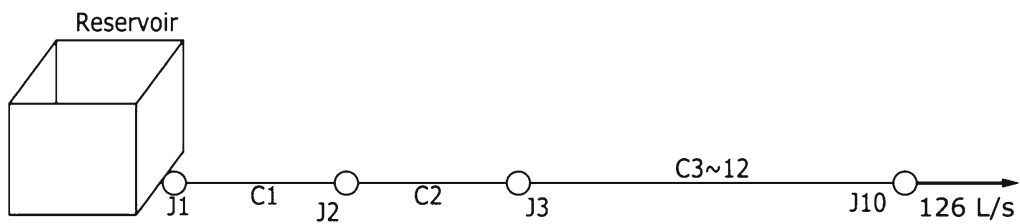

(c)

Figure 3. The different three levels of simplifications for the used pipes network. (a) The first simplification level. (b) The second simplification level (c) The third simplification level.

\section{Results and discussions}

To show the effect of the pipes network simplification on water hammer phenomenon, three scenarios of transient flows on simplified and original networks were simulated and compared. The original network was simplified up to three levels according to both hydraulic and hydraulic plus water quality equivalence, moreover the original network is loaded by either concentrated or distributed water demands at their joints. The transient flow was initialized through linearly and suddenly shut-off or release of concentrated or distributed water demands through a short period of two seconds. WHAMO software which uses the implicit finite difference scheme for solving the momentum and continuity equations at unsteady-state case was used in the simulation.

Three scenarios producing transient flows were performed for both simplified and original networks. In the $1^{\text {st }}$ scenario, a concentrated boundary demand at joint $\mathrm{J} 10$ is linearly decreased from 126 to $0 \mathrm{~L} / \mathrm{s}$ through two seconds period. In the $2^{\text {nd }}$ scenario, a concentrated boundary 
Table 2. Lengths, diameters and friction factors of pipes for the three simplification levels.

\begin{tabular}{|c|c|c|c|c|c|c|c|c|c|c|c|}
\hline & Pipe's ID & & $\mathrm{C} 1$ & $\mathrm{C} 2$ & C3 & $\mathrm{C} 4,5$ & $\mathrm{C} 6,7$ & $\mathrm{C} 8$ & C9,10 & $\mathrm{C} 11$ & $\mathrm{C} 12$ \\
\hline \multirow[t]{6}{*}{ Level 1} & H. Equiv. & $L$ & 305 & 305 & 305 & 610 & 610 & 305 & 610 & 305 & 305 \\
\hline & & $D$ & 300 & 300 & 300 & 164.4 & 164.4 & 250 & 164.4 & 300 & 250 \\
\hline & & $f$ & 0.026 & 0.026 & 0.024 & 0.018 & 0.018 & 0.022 & 0.018 & 0.024 & 0.022 \\
\hline & H. \& Q. Equiv. & $L$ & 305 & 305 & 305 & 610 & 610 & 305 & 610 & 305 & 305 \\
\hline & & $\mathrm{D}$ & 300 & 300 & 300 & 176.8 & 176.8 & 250 & 176.8 & 300 & 250 \\
\hline & & $f$ & 0.026 & 0.026 & 0.024 & 0.026 & 0.026 & 0.022 & 0.026 & 0.024 & 0.022 \\
\hline \multirow[t]{7}{*}{ Level 2} & Pipe’s ID & & $\mathrm{C} 1$ & $\mathrm{C} 2$ & \multicolumn{2}{|c|}{$\mathrm{C} 3,4,5,11$} & $\mathrm{C} 6,7$ & \multicolumn{2}{|c|}{$\mathrm{C} 8,9,10,12$} & & \\
\hline & \multirow[t]{3}{*}{ H. Equiv. } & $L$ & 305 & 305 & 1220 & & 610 & & & & \\
\hline & & $D$ & 300 & 300 & 186.5 & & 164.4 & 183.7 & & & \\
\hline & & $f$ & 0.026 & 0.026 & 0.018 & & 0.018 & 0.018 & & & \\
\hline & \multirow[t]{3}{*}{ H. \& Q. Equiv. } & $L$ & 305 & 305 & 1220 & & 610 & 1220 & & & \\
\hline & & $D$ & 300 & 300 & 246.2 & & 176.8 & 216.5 & & & \\
\hline & & $f$ & 0.026 & 0.026 & 0.072 & & 0.026 & 0.041 & & & \\
\hline \multirow[t]{7}{*}{ Level 3} & \multirow{4}{*}{$\begin{array}{l}\text { Pipe's ID } \\
\text { H. Equiv. }\end{array}$} & & $\mathrm{C} 1$ & $\mathrm{C} 2$ & \multicolumn{2}{|c|}{ C $3 \sim \mathrm{C} 12$} & & & & & \\
\hline & & $L$ & 305 & 305 & \multicolumn{2}{|c|}{610} & & & & & \\
\hline & & $D$ & 300 & 300 & \multicolumn{2}{|l|}{251.8} & & & & & \\
\hline & & $f$ & 0.026 & 0.026 & 0.018 & & & & & & \\
\hline & \multirow[t]{3}{*}{ H. \& Q. Equiv. } & $L$ & 305 & 305 & 610 & & & & & & \\
\hline & & $D$ & 300 & 300 & 504 & & & & & & \\
\hline & & $f$ & 0.026 & 0.026 & 0.536 & & & & & & \\
\hline
\end{tabular}

$-L=$ Pipe length $(m), D=$ Pipe diameter $(\mathrm{mm})$, and $f=$ Friction factor.

$-H$. Equiv. $=$ Hydraulic equivalence, $H . \& Q$. Equiv. $=$ Hydraulic plus quality equivalence

demand at joint $\mathrm{J} 10$ is linearly increased from 0 to $126 \mathrm{~L} / \mathrm{s}$ through two seconds period. Both the first and second scenarios are applied on the original network and three levels of simplified networks according to both hydraulic and hydraulic plus water quality equivalence. In the $3^{\text {rd }}$ scenario, a concentrated boundary demand of $126 \mathrm{~L} /$ sat the endpoint (J10) was distributed equally with a boundary value of $14 \mathrm{~L} / \mathrm{s}$ on nine nodes of the original network $(\mathrm{J} 3 \sim \mathrm{J} 11)$ and both the concentrated and distributed demands were suddenly shut-off or released through a short period of two seconds. The third scenario was applied only on the original network.

\subsection{Effect of suddenly concentrated demand shut-off ( $1^{\text {st }}$ scenario)}

To examine the effect of network simplifications on water hammer phenomenon when a concentrated boundary demand is suddenly shut-off, the valve at node J10was assumed to be linearly closed in a short period of 2 seconds. Before closing the valve, the flow in the network will be at steady-state with the pressure head controlled by friction losses in the pipes, minor losses in the fittings, and the type of the valve and its opened area. When the valve is closed instantaneously the liquid next to the valve comes to a halt. The liquid is then compressed by the liquid upstream which is still flowing. This compression causes a local increase in the pressure of the liquid. The total pressure acting within the pipes equals the summing of the steady-state and the water hammer induced pressure. The walls of the pipes around the fluid are stretched by the resulting excess pressure. A chain reaction then takes place along the lengths of the pipes with each stationary element of fluid being compressed by the flowing fluid upstream. When the pressure wave reaches the reservoir, the fluid in the pipes is now at rest and the pressure cannot exceed 
the boundary water depth in the reservoir, thus water starts to flow out of the pipes into the reservoir. An unloading pressure wave now travels back along the pipes towards the valve. When the unloading wave reaches the valve, the water in the pipes is now flowing out of the pipe into the reservoir, but at the closed valve the water must be at rest. This now causes a negative pressure wave to travel back up the pipes towards the reservoir. When the pressure wave hits the reservoir the flow in the pipes will be at rest, but the pressure head is now below the reservoir level, flow reverses in the pipes and another unloading wave travels back along the pipes towards the valve. A cycle of pressure waves (positive - unloading - negative - unloading) now travels up and down the lengths of the pipes.

The pressure wave travels along the pipes network with a certain velocity, which is called the celerity. The pressure wave's celerity is affected by the modulus of elasticity of fluid and pipes' material, water density, pipes' diameter, and pipes' wall thickness. For instantaneous valve closure the transient increase and decrease in water pressure due to water hammer depend mainly on the celerity of the wave, water density, and water velocity in the pipes under the steady-state conditions.

Figures 4 to 6 show the transient pressure head at node J10 due to its suddenly demand shut-off for the different three levels of simplifications compared with that of the original network through a duration of simulation of 100 seconds just after the valve closure. As shown in each figure, for both simplified and original networks the peak pressure values occur in the first

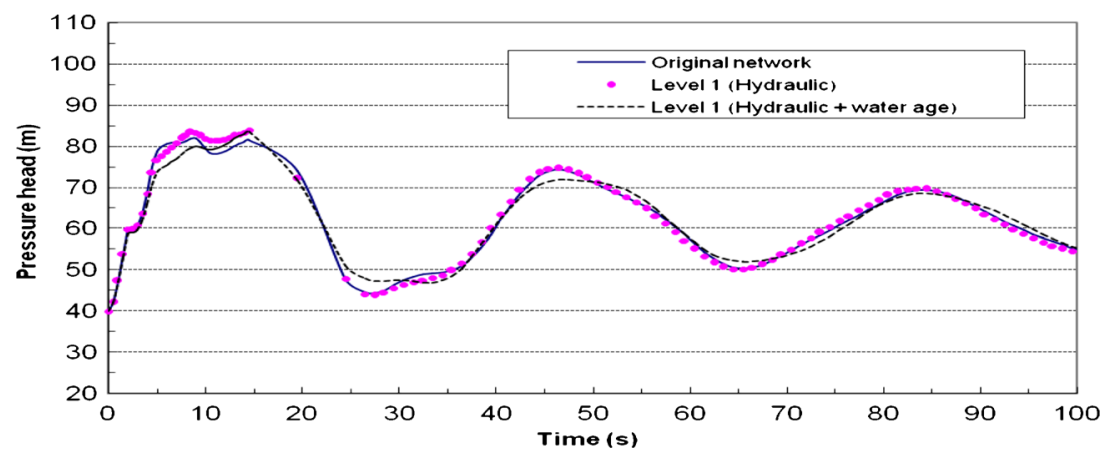

Figure 4. Transient pressure head for the simplified (level 1) and original Networks at node J10 due to its sudden shut-off demand.

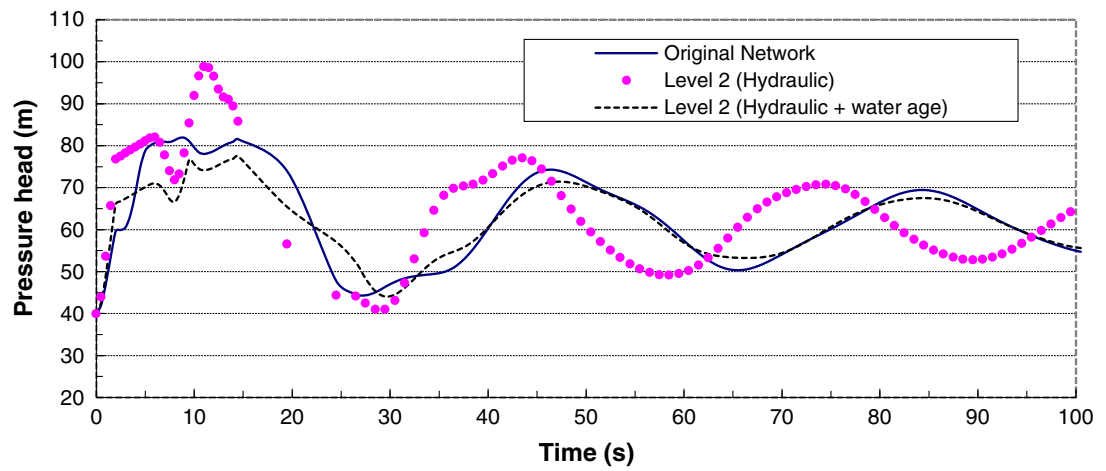

Figure 5. Transient pressure head for the simplified (level 2) and original Networks at node J10 due to its sudden shut-off demand. 


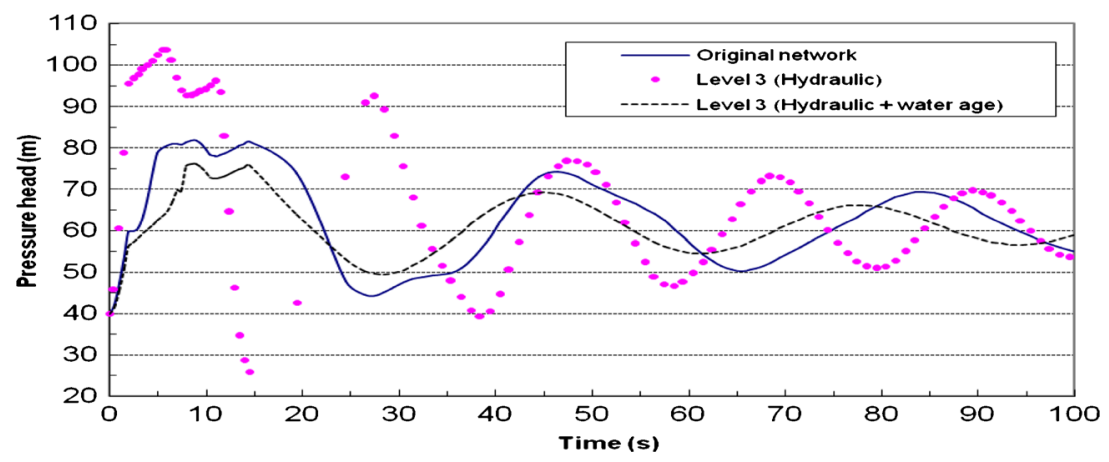

Figure 6. Transient pressure head for the simplified (level 3) and original Networks at node J10 due to its sudden shut-off demand.

cycle thus demonstrate the effect of friction on damping pressure waves. From the figures it is noticeable that, the hydraulic equivalence simplification increases the peak values of the transient pressure head compared with those of the original network, however, the simplification according to hydraulic plus water quality equivalence reduces the peak values.

In comparison between the figures, it is clear that as the level of simplification increases, the transient pressure head of the simplified network deviates more from that of the original network. The figures illustrate that, in the case of the hydraulic equivalence the frequency of the transient pressure waves increases as the simplification level increases. However, the frequencies of the transient pressure waves for the original and hydraulic plus water quality simplified networks are the same. For the hydraulic plus water quality equivalence simplification as the water age is a controlling parameter, the frequencies of the transient pressure waves for both original and simplified networks should have the same trend. In all cases it is clear that, the simulation period (100 seconds) is not sufficient to achieve the steady-state flow conditions in both simplified and original networks.

Just after the valve is suddenly closed, a cycle of pressure waves (positive - unloading negative - unloading) has been induced. Positive and negative waves start at node J10 while the unloading waves start at the reservoir. At the time of the positive and second unloading pressure waves the direction of the transient flow of the liquid remains towards the valve. Through the first unloading and negative pressure waves the liquid reverses towards the reservoir (backflow).

Figures 7 to 9 show the transient flow rate at node J2, as an example, due to suddenly concentrated boundary demand shut-off at node J10 (linearly closed in a short period of 2 seconds) through a duration of simulation of 100 seconds for the different levels of simplifications compared with that of the original network. As shown in the figures at time zero and before closing the valve, the flow was at the steady-state with the boundary flow rate of $126 \mathrm{~L} / \mathrm{s}$. From each figure, it can be seen that the hydraulic equivalence simplification increases the peak values of the transient flow rate compared with those of the original network, however, the simplification according to the hydraulic plus water quality equivalence reduces the peak values. In comparison between these figures, it is noticeable that as the level of simplification increases the transient flow rate of the simplified network deviates more from that of the original network.

As shown in figure 9, the third level of hydraulic equivalence simplification produces a transient flow rate with waves that have high frequency compared with those of hydraulic plus water quality equivalence simplification, original network, and even others hydraulic equivalence simplification levels. 


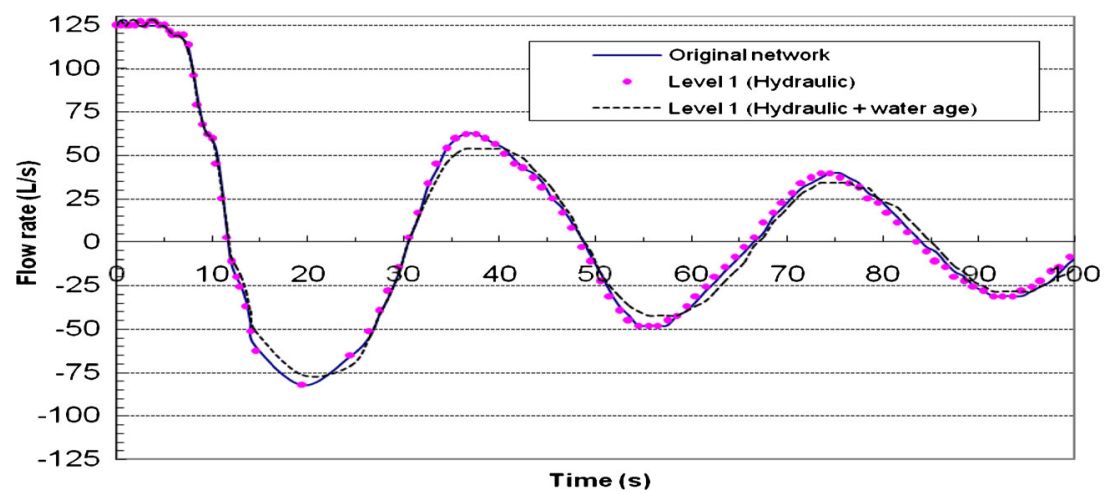

Figure 7. Transient flow rate at node $\mathrm{J} 2$ due to suddenly demand shut-off at node 10 for the simplified (level 1) and original networks.

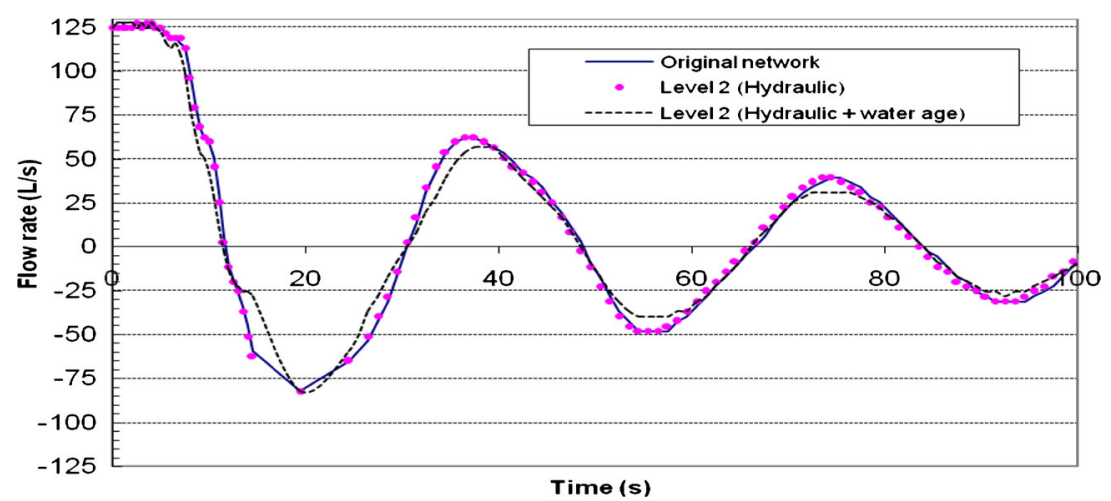

Figure 8. Transient flow rate at node $\mathrm{J} 2$ due to suddenly demand shut-off at node 10 for the simplified (level 2) and original networks.

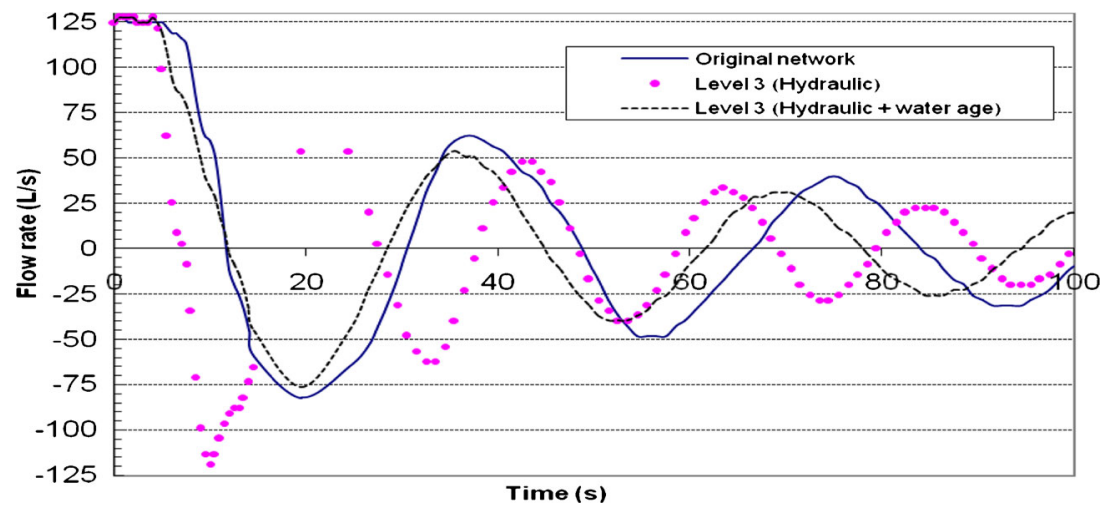

Figure 9. Transient flow rate at node $\mathrm{J} 2$ due to suddenly demand shut-off at node 10 for the simplified (level 3) and original networks. 


\subsection{Effect of suddenly concentrated demand release (2 ${ }^{\text {nd }}$ scenario)}

To show the effect of network simplifications on water hammer phenomenon when a concentrated boundary demand is released suddenly, the valve at node J10 was assumed to be linearly opened in a short period of 2 seconds. When the valve at node J10 is completely closed, there is no-flow in the network; consequently the pressure head through the network equals the water boundary level in the reservoir $(59 \mathrm{~m})$. As the valve at node $\mathrm{J} 10$ is suddenly opened and the demand is released from 0 to a boundary value of $126 \mathrm{~L} / \mathrm{s}$ a negative pressure wave travels along the pipes network from node $\mathrm{J} 10$ towards the reservoir. A cycle of pressure waves (negative unloading -positive - unloading) starts to travel up from node J10 towards the reservoir and down from the reservoir to node $\mathrm{J} 10$ through the pipes in a successive manner.

Figures 10 to 12 illustrate the transient pressure head at node J10 after its suddenly demand release for the three levels of simplifications compared with that of the original network. As shown in each figure, the peaks of the transient pressure waves are gradually damped due to the friction effect. From figures, it is clear that the transient pressure conditions are damped fast within 50 seconds and the steady-state conditions prevail. From each figure, it is noticeable that the hydraulic equivalence simplification increases the peak values of the transient pressure heads compared with those of the original network, however, the simplification according to hydraulic plus water quality equivalence reduces the peak values. In comparison between these figures, it

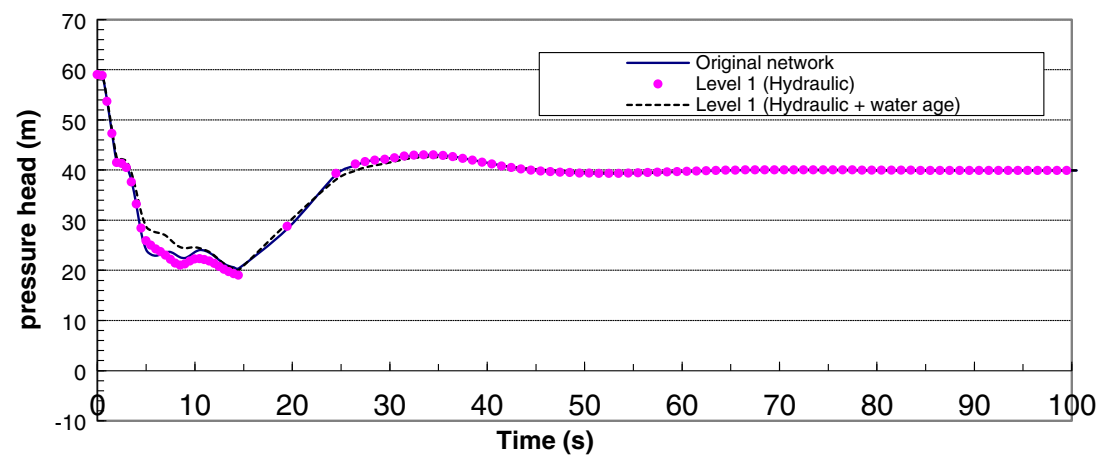

Figure 10. Transient pressure head at node $\mathrm{J} 10$ due to its suddenly demand Release for the simplified (level 1) and original networks.

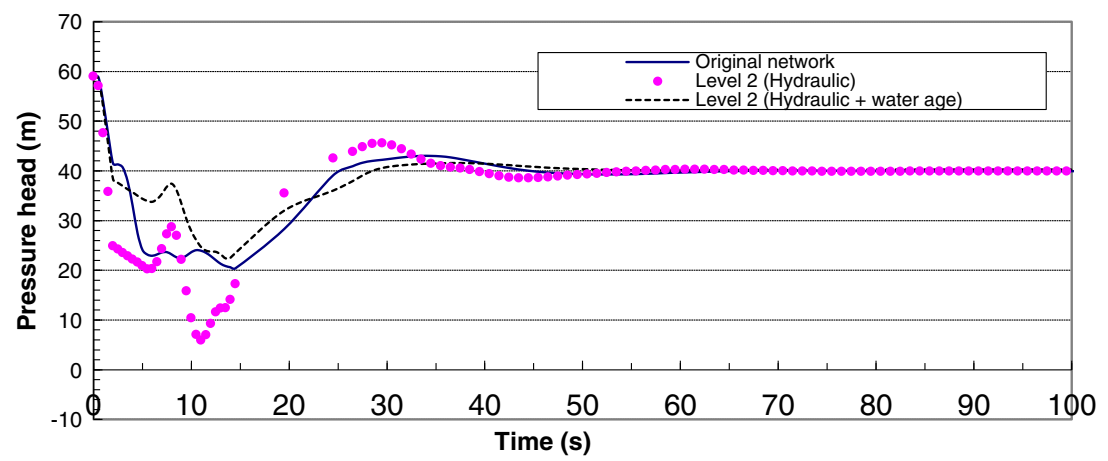

Figure 11. Transient pressure head at node $\mathbf{J} 10$ due to its suddenly demand Release for the simplified (level 2) and original networks. 


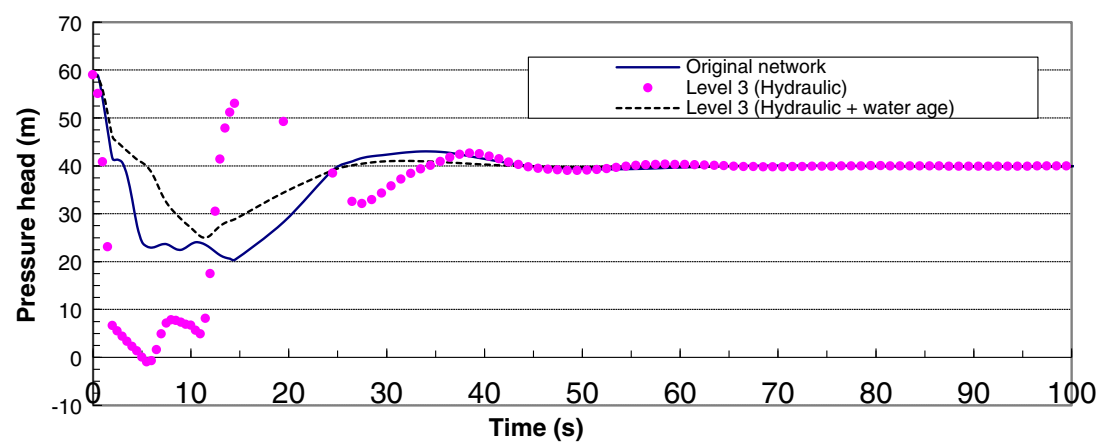

Figure 12. Transient pressure head at node $\mathrm{J} 10$ due to its sudden demand release for the simplified (level 3) and original networks.

is clear that as the level of simplification increases, the transient pressure head of the simplified network deviates more from that of the original network.

Figure 12 illustrates that the frequency of the transient pressure waves for the third level of the hydraulic equivalence simplification is higher than those of the hydraulic plus water quality simplification, original network, and even others hydraulic simplification levels.

Figures 13 to 15 show the transient flow rate at node $\mathrm{J} 2$, as an example, after releasing the demand at node J10 from zero to a boundary value of $126 \mathrm{~L} / \mathrm{s}$ linearly in a short period of two seconds for the different three levels of simplifications compared with that of the original network. In comparison between these figures, it is noticeable that as the level of simplification increases the deviation of the transient flow rate from the original case increases. Also, it can be seen that the hydraulic equivalence simplification increases the peak values of the transient flow rate compared with those of the original network, however, the simplification according to hydraulic plus water quality equivalence reduces the peak values. Also, from the figures, it is clear that the transient flow conditions are damped fast within 50 seconds and the steady-state conditions prevail.

In general, figures 10 to 15 demonstrate that the transient flow rate at node $\mathrm{J} 2$ for the different levels of simplifications has an inverse trend to that of the transient pressure head at node J10 with a short time lag which could be attributed to the location's difference between the concerned nodes.

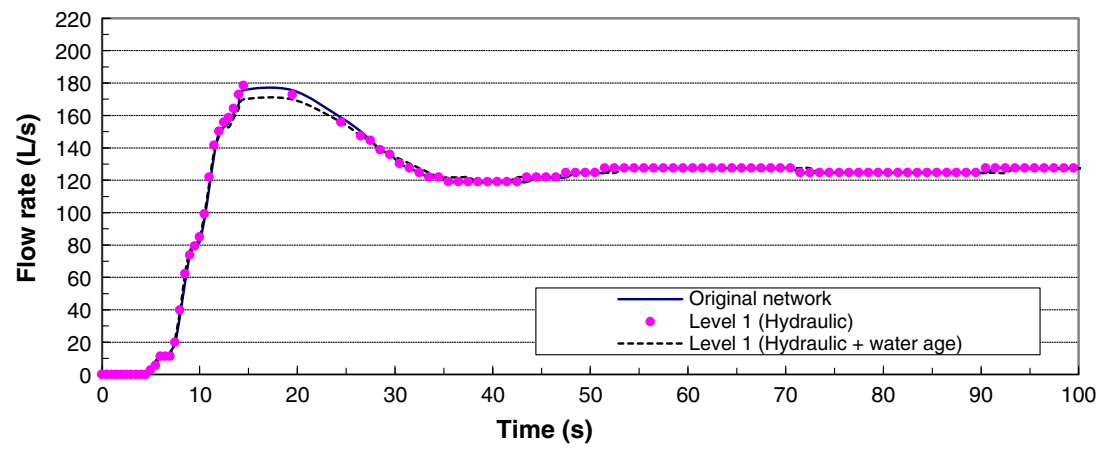

Figure 13. Transient flow rate at node $\mathrm{J} 2$ due to sudden demand releases at node $\mathrm{J} 10$ for the simplified (level 1) and original networks. 


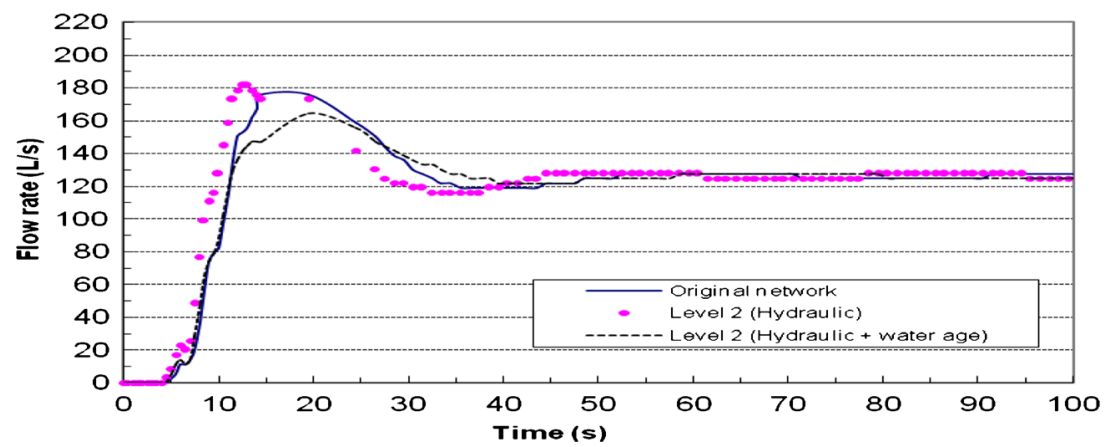

Figure 14. Transient flow rate at node $\mathrm{J} 2$ due to sudden demand releases at node $\mathrm{J} 10$ for the simplified (level 2) and original networks.

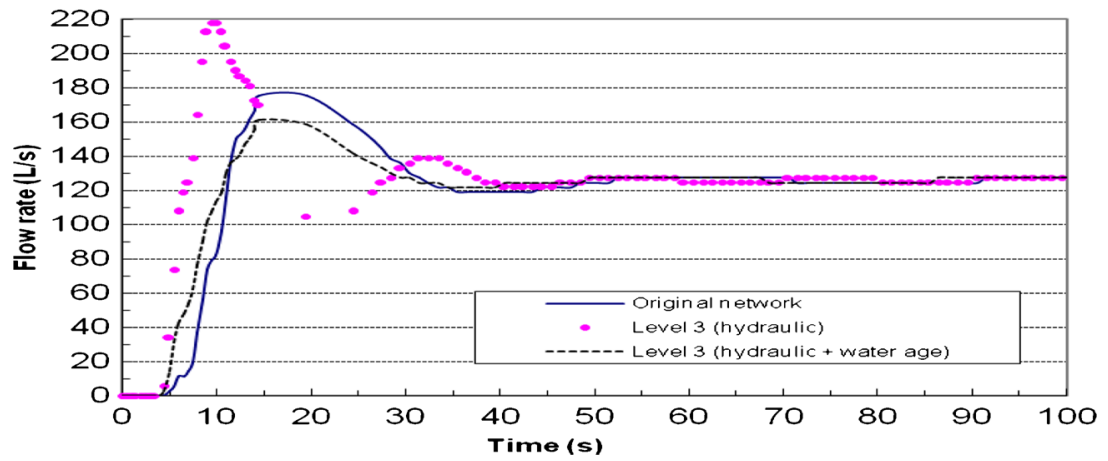

Figure 15. Transient flow rate at node $\mathrm{J} 2$ due to sudden demand releases at node $\mathrm{J} 10$ for the simplified (level 3) and original networks.

\subsection{Effect of water demands' concentration ( $3^{\text {rd }}$ scenario)}

To show the effect of water demands' concentration on water hammer phenomenon, a boundary distributed demands loaded on nine nodes of the original network (J3 J11) with a value of $14 \mathrm{~L} / \mathrm{s}$ at each node were concentrated at the end node (J10) with an equivalent boundary value of $126 \mathrm{~L} / \mathrm{s}$ and both distributed and concentrated demands were suddenly and linearly shut-off and released through a short period of 2 seconds. Figures 16 and 17 show the simulated transient pressure head at node $\mathrm{J} 10$ and flow rate at node $\mathrm{J} 2$ for both distributed and concentrated demands for two cases of suddenly shut-off and release, respectively. It is clear from these figures that concentrating the demands produces bigger transient pressure head and flow rate compared with the distributed one in case of demands shut-off as well as demands release. It is observed from the figures that 100 seconds period after shutting-off the concentrated or distributed demands is not sufficient to reach to the steady-state flow while it takes only around 50 seconds after releasing the demands to reach to the steady-state conditions. Also, figures 16 and 17 illustrate that the transient pressure head at node $\mathrm{J} 10$ and flow rate at node $\mathrm{J} 2$ for the original network loaded by either distributed or concentrated demands have an inverse trend with a short time lag which may be attributed to the location's difference between the two joints.

To confirm the obtained results, pump shut-off effect on transient flow at different simplification levels is shown in figure 18 , where the transient head at node $\mathbf{J} 2$ was drawn versus time for 


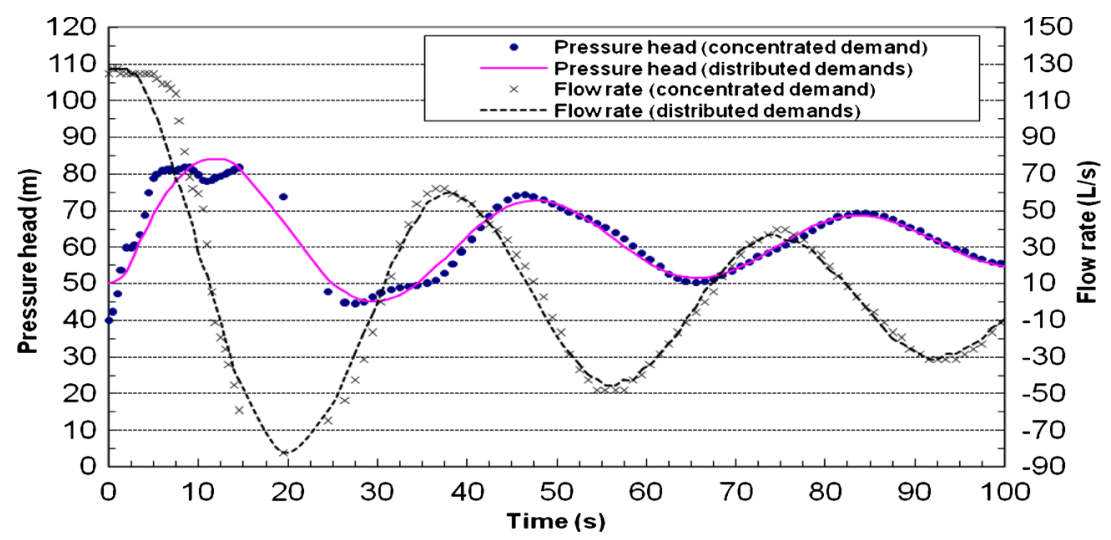

Figure 16. Transient pressure head at node $\mathrm{J} 10$ and flow rate at node $\mathrm{J} 2$ due to concentrated and distributed demands sudden shut-off.

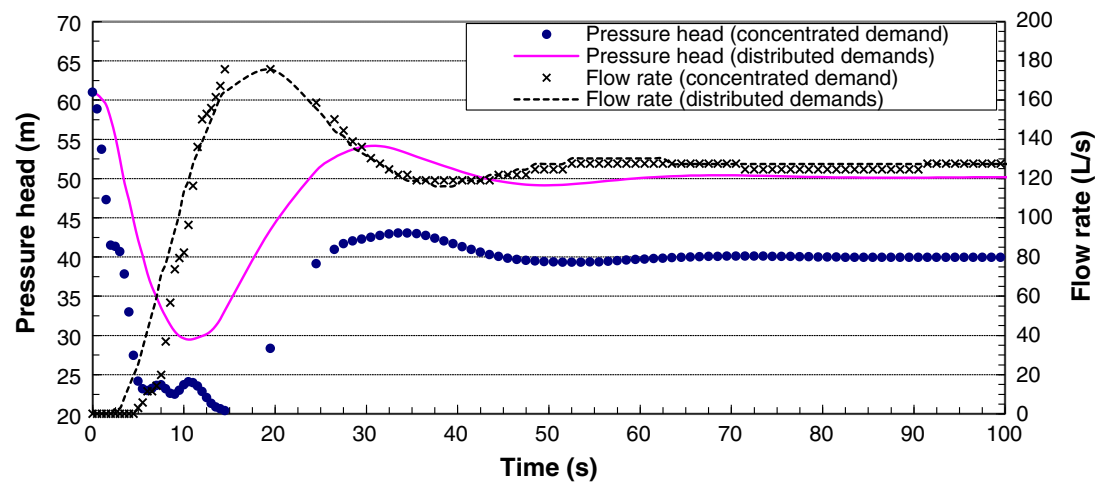

Figure 17. Transient pressure head at node $\mathrm{J} 10$ and flow rate at node $\mathrm{J} 2$ due to sudden release of concentrated and distributed demands.

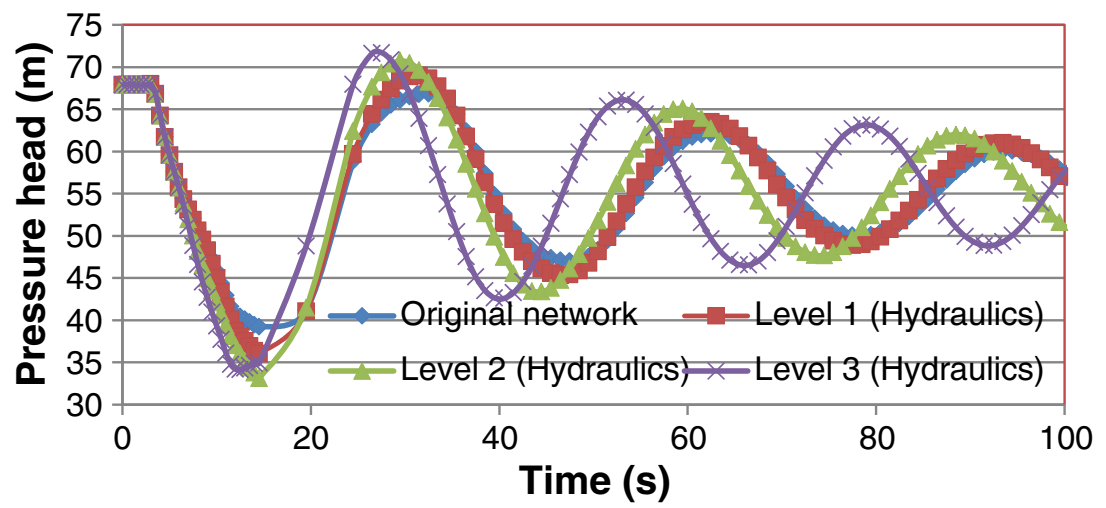

Figure 18. Transient pressure head at node $\mathrm{J} 2$ due to pump shut-off for different simplified levels. 
original network and the three levels of simplification. It can be shown from this figure that the maximum pressure increases by increasing simplification degree and also the wave frequency.

\section{Conclusions}

Simplification is an indispensible design step for water supply and irrigation pipes networks. Three types of simplifications may be performed on distribution networks as; hydraulic equivalence, hydraulic plus water quality equivalence, and demands' concentration. Variable water demands and usage patterns in water distribution systems may create transient flow that could make so many undesirable consequences. The effect of pipes networks' simplification on the transient flow must be accurately determined so that they can be properly designed. Three scenarios producing transient flow in both simplified and original networks were investigated in this research. The transient flow was initialized by linearly and suddenly shutting-off or releasing of either distributed or concentrated boundary demands in a short period of 2 seconds. WHAMO software which uses the implicit finite difference scheme for solving the momentum and continuity equations at unsteady-state case was used in the simulation. The major findings of this study can be summarized as follows.

(i) In all cases, for both simplified and original networks the peaks of the transient pressure and flow rate occur in the first cycle thus demonstrate the effect of friction on damping the transient flow.

(ii) Hydraulic equivalence and demands' concentration simplifications increase the peak values for the transient pressure and flow rate in the simplified network compared with the original one. However, hydraulic plus water quality equivalence simplification results in an adverse effect.

(iii) As the degree of simplification increases the transient pressure head and flow rate of the simplified network deviate more from those of the original network.

(iv) In case of the hydraulic equivalence, the frequency of the transient waves increase as the simplification level increases. However, the frequencies of the original and hydraulic plus water quality simplified networks are found to be the same. This result is quite clear in the $3^{\text {rd }}$ level of hydraulic simplification which converts the pipes network from looped to a single line.

(v) For the transient flow results from boundary demands' shutting-off, the simulation period (100 seconds) is not sufficient to achieve the steady-state flow conditions in both simplified and original networks simulated in this study. However, it takes only around 50 seconds after releasing the boundary demands to reach to the steady-state flow conditions.

\section{References}

Abd El-Gawad S M 1994 Water hammer analysis for the pipeline Ahmed Hamdi Tunnel, Abu-Radis. Eng. Res. J. 6: 40-54

Ali N A, Mohamed H I, El-Darder M E and Mohame A A 2010 Analysis of transient flow phenomenon in pressurized pipes system and methods of protection. J. Eng. Sci. Assiut University 38(2): 323-342

Al-Khomairi A M 2005 Use of the steady-state orifice equation in the computation of transient flow through pipe leaks. The Arabian J. Sci. Eng. 30(IB): 33-45

Batterton S 2006 Water hammer: An analysis of plumbing systems, intrusion, and pump operation. Thesis submitted to the Faculty of The Virginia Polytechnic Institute and State Univ. in partial fulfillment of the requirements for the degree of M. Sc. in Civil Eng., pp. 147 
Chaudhry H M and Yevjevich V 1981 Closed-Conduit flow. Water Resources Publications, Littleton, U.S.A Fitzgerald R and Van Blaricum V L 1998 Water hammer and mass oscillation (WHAMO) 3.0 user's manual Fouzi A and Ali F 2001 Comparative study of the phenomenon of propagation of elastic waves in conduits. Proceed. of The World Congress on Eng., Vol. III, London, U.K

Jönnsson L 1999 Hydraulic transient as a monitoring device. XXVII IAHR Congress, Graz, Austria

Jung B S, Boulos P F and Wood D J 2007 Pitfalls of water distribution model skeletonization for surge analysis. J. AWWA 99(12): 87-98

Jung B S, Boulos P F and Wood D J 2009 Effect of pressure-sensitive demand on surge analysis. J. AWWA 101(4): 100-111

Kwon H J 2007 Computer simulations of transient flow in a real city water distribution system, KSCE. J. Civil Eng. 11(1): 43-49

Mohamed H I 2003 Parametric study for the water hammer phenomenon in pipelines. $1^{\text {st }}$ Int. Conf. of Civil Eng. Sci. ICCESI, Vol. 2, pp. 1-12, Assiut, Egypt

Mohamed H I and Ahmed S S 2011 Effect of simplifying the water supply pipe networks on water quality simulation. Inter. Confer. for Water, Energy Environ., Sharijah, UAE, pp. 41-46

Raczynski A, Kirkpatrick W, Rehnstrom D, Boulos P and Lansey K 2008 Developing hydraulic and water quality equivalent systems. Proceed. of the $10^{\text {th }}$ Annual Water Distr. Systems Conf., WDSA2008, Krugar National Park, South Africa

Ramos H, Covas D, Borga A and Loureiro A 2004 A surge damping analysis in pipe systems: Modeling and experiments. 42(4): 413-425

Samani H M V and Khayatzadeh A 2002 Transient flow in pipe networks. J. Hyd. Res. 40(5): 637-644

Simpson A R and Wu Z Y 1997 Computer modelling of hydraulic transient in pipe networks and associated design criteria. MODSIM97, International Congress on Modelling and Simulation, Modelling and Simulation Society of Australia, Hobart, Tasmania, Australia

Stephenson D 2002 Simple guide for design of air vessels for water hammer protection of pumping lines. J. Hyd. Eng. 128(8): 792-797

Yang K 2001 Practical method to prevent liquid column separation. J. Hyd. Eng. 127(7): 620-623 